\title{
Using Confidence Bands for Parallel Texts Alignment
}

\author{
António RIBEIRO \\ Departamento de Informática \\ Faculdade de Ciências e Tecnologia \\ Universidade Nova de Lisboa \\ Quinta da Torre \\ P-2825-114 Monte da Caparica \\ Portugal \\ ambar@di.fct.unl.pt
}

\author{
Gabriel LOPES \\ Departamento de Informática \\ Faculdade de Ciências e Tecnologia \\ Universidade Nova de Lisboa \\ Quinta da Torre \\ P-2825-114 Monte da Caparica \\ Portugal \\ gpl@di.fct.unl.pt
}

\author{
João MEXIA \\ Departamento de Matemática \\ Faculdade de Ciências e Tecnologia \\ Universidade Nova de Lisboa \\ Quinta da Torre \\ P-2825-114 Monte da Caparica \\ Portugal
}

\begin{abstract}
This paper describes a language independent method for alignment of parallel texts that makes use of homograph tokens for each pair of languages. In order to filter out tokens that may cause misalignment, we use confidence bands of linear regression lines instead of heuristics which are not theoretically supported. This method was originally inspired on work done by Pascale Fung and Kathleen McKeown, and Melamed, providing the statistical support those authors could not claim.
\end{abstract}

\section{Introduction}

Human compiled bilingual dictionaries do not cover every term translation, especially when it comes to technical domains. Moreover, we can no longer afford to waste human time and effort building manually these ever changing and incomplete databases or design language specific applications to solve this problem. The need for an automatic language independent task for equivalents extraction becomes clear in multilingual regions like Hong Kong, Macao, Quebec, the European Union, where texts must be translated daily into eleven languages, or even in the U.S.A. where Spanish and English speaking communities are intermingled.

Parallel texts (texts that are mutual translations) are valuable sources of information for bilingual lexicography. However, they are not of much use unless a computational system may find which piece of text in one language corresponds to which piece of text in the other language. In order to achieve this, they must be aligned first, i.e. the various pieces of text must be put into correspondence. This makes the translations extraction task easier and more reliable. Alignment is usually done by finding correspondence points - sequences of characters with the same form in both texts (homographs, e.g. numbers, proper names, punctuation marks), similar forms (cognates, like Region and Região in English and Portuguese, respectively) or even previously known translations.

Pascale Fung and Kathleen McKeown (1997) present an alignment algorithm that uses term translations as correspondence points between English and Chinese. Melamed (1999) aligns texts using correspondence points taken either from orthographic cognates (Michel Simard et al., 1992) or from a seed translation lexicon. However, although the heuristics both approaches use to filter noisy points may be intuitively quite acceptable, they are not theoretically supported by Statistics.

The former approach considers a candidate correspondence point reliable as long as, among some other constraints, "[...] it is not too far away from the diagonal [...]" (Pascale Fung and Kathleen McKeown, 1997, p.72) of a rectangle whose sides sizes are proportional to the lengths of the texts in each language (henceforth, 'the golden translation diagonal'). The latter approach uses other filtering parameters: maximum point ambiguity level, point dispersion and angle deviation (Melamed, 1999, pp. 115-116).

António Ribeiro et al. (2000a) propose a method to filter candidate correspondence points generated from homograph words which occur only once in parallel texts (hapaxes) using linear regressions and statistically supported noise filtering methodologies. The method avoids heuristic filters and they claim high precision alignments. 
In this paper, we will extend this work by defining a linear regression line with all points generated from homographs with equal frequencies in parallel texts. We will filter out those points which lie outside statistically defined confidence bands (Thomas Wonnacott and Ronald Wonnacott, 1990). Our method will repeatedly use a standard linear regression line adjustment technique to filter unreliable points until there is no misalignment. Points resulting from this filtration are chosen as correspondence points.

The following section will discuss related work. The method is described in section 2 and we will evaluate and compare the results in section 3. Finally, we present conclusions and future work.

\section{Background}

There have been two mainstreams for parallel text alignment. One assumes that translated texts have proportional sizes; the other tries to use lexical information in parallel texts to generate candidate correspondence points. Both use some notion of correspondence points.

Early work by Peter Brown et al. (1991) and William Gale and Kenneth Church (1991) aligned sentences which had a proportional number of words and characters, respectively. Pairs of sentence delimiters (full stops) were used as candidate correspondence points and they ended up being selected while aligning. However, these algorithms tended to break down when sentence boundaries were not clearly marked. Full stops do not always mark sentence boundaries, they may not even exist due to OCR noise and languages may not share the same punctuation policies.

Using lexical information, Kenneth Church (1993) showed that cheap alignment of text segments was still possible exploiting orthographic cognates (Michel Simard et al., 1992), instead of sentence delimiters. They became the new candidate correspondence points. During the alignment, some were discarded because they lied outside an empirically estimated bounded search space, required for time and space reasons.

Martin Kay and Martin Röscheisen (1993) also needed clearly delimited sentences. Words with similar distributions became the candidate correspondence points. Two sentences were aligned if the number of correspondence points associating them was greater than an empirically defined threshold: "[...] more than some minimum number of times [...]" (Martin Kay and Martin Röscheisen, 1993, p.128). In Ido Dagan et al. (1993) noisy points were filtered out by deleting frequent words.

Pascale Fung and Kathleen McKeown (1994) dropped the requirement for sentence boundaries on a case-study for English-Chinese. Instead, they used vectors that stored distances between consecutive occurrences of a word (DK-vec's). Candidate correspondence points were identified from words with similar distance vectors and noisy points were filtered using some heuristics. Later, in Pascale Fung and Kathleen McKeown (1997), the algorithm used extracted terms to compile a list of reliable pairs of translations. Those pairs whose distribution similarity was above a threshold became candidate correspondence points (called potential anchor points). These points were further constrained not to be "too far away" from the 'translation diagonal'.

Michel Simard and Pierre Plamondon (1998) aligned sentences using isolated cognates as candidate correspondence points, i.e. cognates that were not mistaken for others within a text window. Some were filtered out if they either lied outside an empirically defined search space, named a corridor, or were "not in line" with their neighbours.

Melamed (1999) also filtered candidate correspondence points obtained from orthographic cognates. A maximum point ambiguity level filters points outside a search space, a maximum point dispersion filters points too distant from a line formed by candidate correspondence points and a maximum angle deviation filters points that tend to slope this line too much.

Whether the filtering of candidate correspondence points is done prior to alignment or during it, we all want to find reliable correspondence points. They provide the basic means for extracting reliable information from parallel texts. However, as far as we learned from the above papers, current methods have repeatedly used statistically unsupported heuristics to filter out noisy points. For instance, the 'golden translation diagonal' is mentioned in all of them but none attempts filtering noisy points using statistically defined confidence bands. 


\section{Correspondence Points Filters}

\subsection{Overview}

The basic insight is that not all candidate correspondence points are reliable. Whatever heuristics are taken (similar word distributions, search corridors, point dispersion, angle deviation,...), we want to filter the most reliable points. We assume that reliable points have similar characteristics. For instance, they tend to gather somewhere near the 'golden translation diagonal'. Homographs with equal frequencies may be good alignment points.

\subsection{Source Parallel Texts}

We worked with a mixed parallel corpus consisting of texts selected at random from the Official Journal of the European Communities ${ }^{1}$ (ELRA, 1997) and from The Court of Justice of the European Communities ${ }^{2}$ in eleven languages ${ }^{3}$.

\begin{tabular}{ccccc}
\multicolumn{5}{c}{ Sub-corpus } \\
\hline Language & Written Questions & Debates & Judgements & Total \\
\hline $\mathrm{da}$ & $259 \mathrm{k}(52 \mathrm{k})$ & $2,0 \mathrm{M}(395 \mathrm{k})$ & $16 \mathrm{k}(3 \mathrm{k})$ & $2250 \mathrm{k}$ \\
$\mathrm{de}$ & $234 \mathrm{k}(47 \mathrm{k})$ & $1,8 \mathrm{M}(368 \mathrm{k})$ & $15 \mathrm{k}(3 \mathrm{k})$ & $2088 \mathrm{k}$ \\
$\mathrm{el}$ & $272 \mathrm{k}(54 \mathrm{k})$ & $1,9 \mathrm{M}(387 \mathrm{k})$ & $16 \mathrm{k}(3 \mathrm{k})$ & $2222 \mathrm{k}$ \\
$\mathrm{en}$ & $263 \mathrm{k}(53 \mathrm{k})$ & $2,1 \mathrm{M}(417 \mathrm{k})$ & $16 \mathrm{k}(3 \mathrm{k})$ & $2364 \mathrm{k}$ \\
$\mathrm{es}$ & $292 \mathrm{k}(58 \mathrm{k})$ & $2,2 \mathrm{M}(439 \mathrm{k})$ & $18 \mathrm{k}(4 \mathrm{k})$ & $2507 \mathrm{k}$ \\
$\mathrm{fi}$ & --- & -- & $13 \mathrm{k}(3 \mathrm{k})$ & $13 \mathrm{k}$ \\
$\mathrm{fr}$ & $310 \mathrm{k}(62 \mathrm{k})$ & $2,2 \mathrm{M}(447 \mathrm{k})$ & $19 \mathrm{k}(4 \mathrm{k})$ & $2564 \mathrm{k}$ \\
$\mathrm{it}$ & $279 \mathrm{k}(56 \mathrm{k})$ & $1,9 \mathrm{M}(375 \mathrm{k})$ & $17 \mathrm{k}(3 \mathrm{k})$ & $2171 \mathrm{k}$ \\
$\mathrm{nl}$ & $275 \mathrm{k}(55 \mathrm{k})$ & $2,1 \mathrm{M}(428 \mathrm{k})$ & $16 \mathrm{k}(3 \mathrm{k})$ & $2431 \mathrm{k}$ \\
$\mathrm{pt}$ & $284 \mathrm{k}(57 \mathrm{k})$ & $2,1 \mathrm{M}(416 \mathrm{k})$ & $17 \mathrm{k}(3 \mathrm{k})$ & $2381 \mathrm{k}$ \\
$\mathrm{sv}$ & --- & --- & $15 \mathrm{k}(3 \mathrm{k})$ & $15 \mathrm{k}$ \\
\hline Total & $2468 \mathrm{k}(55 \mathrm{k})$ & $18,4 \mathrm{M}(408 \mathrm{k})$ & $177 \mathrm{k}(3 \mathrm{k})$ & $21005 \mathrm{k}$ \\
\hline
\end{tabular}

Table 1: Words per sub-corpus (average per text inside brackets; markups discarded $)^{4}$.

For each language, we included:

- five texts with Written Questions asked by members of the European Parliament to the European Commission and their corresponding answers (average: about 60k words or 100 pages / text);

1 Danish (da), Dutch (nl), English (en), French (fr), German (de), Greek (el), Italian (it), Portuguese (pt) and Spanish (es).

${ }^{2}$ Webpage address: curia.eu.int

3 The same languages as those in footnote 1 plus Finnish (fi) and Swedish (sv).

${ }^{4}$ No Written Questions and Debates texts for Finnish and Swedish are available in ELRA (1997) since the texts provided are from the 1992-4 period and it was not until 1995 that the respective countries became part of the European Union.
- five texts with records of Debates in the European Parliament (average: about 400k words or more than 600 pages / text). These are written transcripts of oral discussions;

- five texts with judgements of The Court of Justice of the European Communities (average: about $3 \mathrm{k}$ words or 5 pages / text).

In order to reduce the number of possible pairs of parallel texts from 110 sets (11 languages $\times 10$ ) to a more manageable size of 10 sets, we decided to take Portuguese as the kernel language of all pairs.

\subsection{Generating Candidate Correspon- dence Points}

We generate candidate correspondence points from homographs with equal frequencies in two parallel texts. Homographs, as a naive and particular form of cognate words, are likely translations (e.g. Hong Kong in various European languages). Here is a table with the percentages of occurrences of these words in the used texts:

\begin{tabular}{ccccc}
\multicolumn{5}{c}{ Sub-corpus } \\
\hline Pair & Written Questions & Debates & Judgements & Average \\
\hline pt-da & $2,8 \mathrm{k}(4,9 \%)$ & $2,5 \mathrm{k}(0,6 \%)$ & $0,3 \mathrm{k}(8,1 \%)$ & $2,5 \mathrm{k}(1,1 \%)$ \\
pt-de & $2,7 \mathrm{k}(5,1 \%)$ & $4,2 \mathrm{k}(1,0 \%)$ & $0,4 \mathrm{k}(7,9 \%)$ & $4,0 \mathrm{k}(1,5 \%)$ \\
pt-el & $2,3 \mathrm{k}(4,0 \%)$ & $1,9 \mathrm{k}(0,5 \%)$ & $0,3 \mathrm{k}(6,9 \%)$ & $1,9 \mathrm{k}(0,8 \%)$ \\
pt-en & $2,7 \mathrm{k}(4,8 \%)$ & $2,8 \mathrm{k}(0,7 \%)$ & $0,3 \mathrm{k}(6,2 \%)$ & $2,7 \mathrm{k}(1,1 \%)$ \\
pt-es & $4,1 \mathrm{k}(7,1 \%)$ & $7,8 \mathrm{k}(1,9 \%)$ & $0,7 \mathrm{k}(15,2 \%)$ & $7,4 \mathrm{k}(2,5 \%)$ \\
pt-fi & --- & --- & $0,2 \mathrm{k}(5,2 \%)$ & $0,2 \mathrm{k}(5,2 \%)$ \\
pt-fr & $2,9 \mathrm{k}(5,0 \%)$ & $5,1 \mathrm{k}(1,2 \%)$ & $0,4 \mathrm{k}(9,4 \%)$ & $4,8 \mathrm{k}(1,6 \%)$ \\
pt-it & $3,1 \mathrm{k}(5,5 \%)$ & $5,4 \mathrm{k}(1,3 \%)$ & $0,4 \mathrm{k}(9,6 \%)$ & $5,2 \mathrm{k}(1,8 \%)$ \\
pt-nl & $2,6 \mathrm{k}(4,5 \%)$ & $4,9 \mathrm{k}(1,2 \%)$ & $0,3 \mathrm{k}(8,3 \%)$ & $4,7 \mathrm{k}(1,6 \%)$ \\
pt-sv & --- & --- & $0,3 \mathrm{k}(6,9 \%)$ & $0,3 \mathrm{k}(6,9 \%)$ \\
\hline Average & $2,9 \mathrm{k}(5,1 \%)$ & $4,4 \mathrm{k}(1,1 \%)$ & $0,4 \mathrm{k}(8,4 \%)$ & $4,2 \mathrm{k}(1,5 \%)$ \\
\hline
\end{tabular}

Table 2: Average number of homographs with equal frequencies per pair of parallel texts (average percentage of homographs inside brackets).

For average size texts (e.g. the Written Questions), these words account for about $5 \%$ of the total (about $3 \mathrm{k}$ words / text). This number varies according to language similarity. For instance, on average, it is higher for Portuguese-Spanish than for Portuguese-English.

These words end up being mainly numbers and names. Here are a few examples from a parallel Portuguese-English text: 2002 (numbers, dates), ASEAN (acronyms), Patten (proper names), China (countries), Manila (cities), apartheid (foreign words), Ltd (abbreviations), habitats (Latin words), ferry (common names), global (common vocabulary).

In order to avoid pairing homographs that are not equivalent (e.g. 'a', a definite article in Portuguese and an indefinite article in English), we 
restricted ourselves to homographs with the same frequencies in both parallel texts. In this way, we are selecting words with similar distributions. Actually, equal frequency words helped Jean-François Champollion to decipher the Rosetta Stone for there was a name of a King (Ptolemy V) which occurred the same number of times in the 'parallel texts' of the stone.

Each pair of texts provides a set of candidate correspondence points from which we draw a line based on linear regression. Points are defined using the co-ordinates of the word positions in each parallel text. For example, if the first occurrence of the homograph word Patten occurs at word position 125545 in the Portuguese text and at 135787 in the English parallel text, then the point co-ordinates are (125545,135787). The generated points may adjust themselves well to a linear regression line or may be dispersed around it. So, firstly, we use a simple filter based on the histogram of the distances between the expected and real positions. After that, we apply a finer-grained filter based on statistically defined confidence bands for linear regression lines.

We will now elaborate on these filters.

\subsection{Eliminating Extreme Points}

The points obtained from the positions of homographs with equal frequencies are still prone to be noisy. Here is an example:

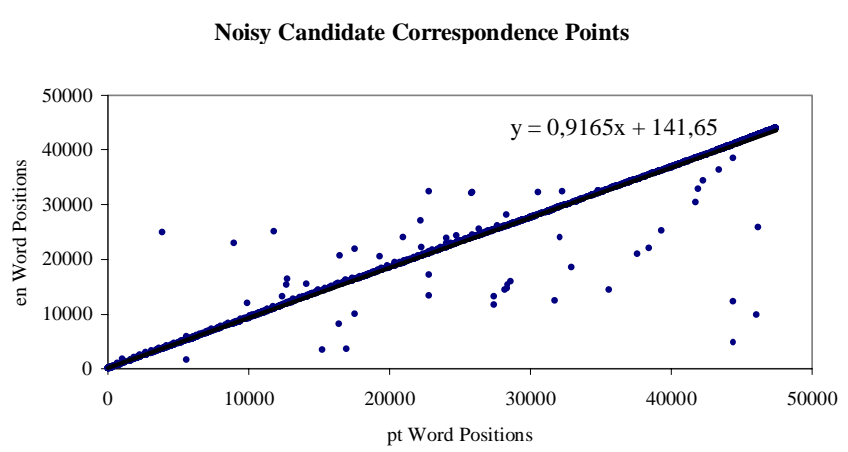

Figure 1: Noisy versus 'well-behaved' ('in line') candidate correspondence points. The linear regression line equation is shown on the top right corner.

The figure above shows noisy points because their respective homographs appear in positions quite apart. We should feel reluctant to accept distant pairings and that is what the first filter does. It filters out those points which are clearly too far apart from their expected positions to be considered as reliable correspondence points.

We find expected positions building a linear regression line with all points, and then determining the distances between the real and the expected word positions:

\begin{tabular}{cl|cc|c}
\multicolumn{2}{c|}{ pt } & \multicolumn{2}{|c|}{ en Positions } & \\
\hline Position & Word & Real & Expected & Distance \\
\hline 3877 & I & 24998 & 3695 & 21303 \\
9009 & etc & 22897 & 8399 & 14499 \\
11791 & I & 25060 & 10948 & 14112 \\
15248 & As & 3398 & 14117 & 10719 \\
16965 & As & 3591 & 15690 & 12099 \\
22819 & volume & 32337 & 21056 & 11281 \\
\hline
\end{tabular}

Table 3: A sample of the distances between expected and real positions of noisy points in Figure 1.

Expected positions are computed from the linear regression line equation $y=a x+b$, where $a$ is the line slope and $b$ is the $\mathrm{Y}$-axis intercept (the value of $y$ when $x$ is 0 ), substituting $x$ for the Portuguese word position. For Table 3, the expected word position for the word $I$ at pt word position 3877 is $0.9165 \times 3877+141.65=3695$ (see the regression line equation in Figure 1) and, thus, the distance between its expected and real positions is $|3695-24998|=21303$.

If we draw a histogram ranging from the smallest to the largest distance, we get:

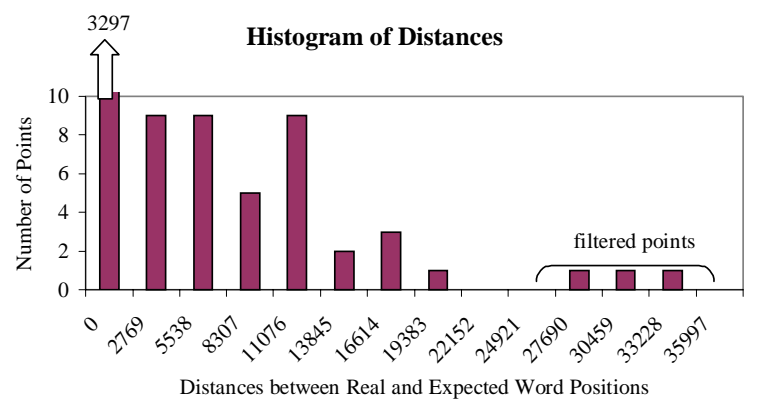

Figure 2: Histogram of the distances between expected and real word positions.

In order to build this histogram, we use the Sturges rule (see 'Histograms' in Samuel Kotz et al. 1982). The number of classes (bars or bins) is given by $1+\log _{2} n$, where $n$ is the total number of points. The size of the classes is given by (maximum distance - minimum distance) / number of classes. For example, for Figure 1, we have 3338 points and the distances between expected and real positions range from 0 to 
35997. Thus, the number of classes is $1+\log _{2} 3338 \cong 12.7 \rightarrow 13$ and the size of the classes is $(35997-0) / 13 \cong 2769$. In this way, the first class ranges from 0 to 2769 , the second class from 2769 to 5538 and so forth.

With this histogram, we are able to identify those words which are too far apart from their expected positions. In Figure 2, the gap in the histogram makes clear that there is a discontinuity in the distances between expected and real positions. So, we are confident that all points above 22152 are extreme points. We filter them out of the candidate correspondence points set and proceed to the next filter.

\subsection{Confidence Bands of Linear Regres- sion Lines}

Confidence bands of linear regression lines (Thomas Wonnacott and Ronald Wonnacott, 1990 , p. 384) help us to identify reliable points, i.e. points which belong to a regression line with a great confidence level (99.9\%). The band is typically wider in the extremes and narrower in the middle of the regression line.

The figure below shows an example of filtering using confidence bands:

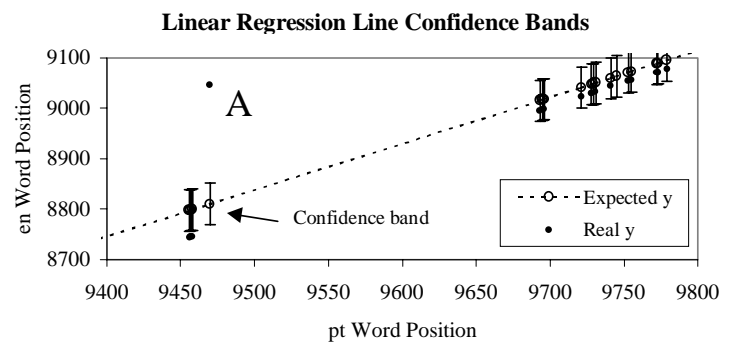

Figure 3: Detail of the filter based on confidence bands. Point A lies outside the confidence band. It will be filtered out.

We start from the regression line defined by the points filtered with the Histogram technique, described in the previous section, and then we calculate the confidence band. Points which lie outside this band are filtered out since they are credited as too unreliable for alignment (e.g. Point A in Figure 3). We repeat this step until no pieces of text belong to different translations, i.e. until there is no misalignment.

The confidence band is the error admitted at an $x$ co-ordinate of a linear regression line. A point $(x, y)$ is considered outside a linear regression line with a confidence level of $99.9 \%$ if its $y$ co-ordinate does not lie within the confidence interval [ $a x+b-\operatorname{error}(x) ; a x+b+\operatorname{error}(x)]$, where $a x+b$ is the linear regression line equation and error $(x)$ is the error admitted at the $x$ co-ordinate. The upper and lower limits of the confidence interval are given by the following equation (see Thomas Wonnacott \& Ronald Wonnacott, 1990, p. 385):

$$
y=(a x+b) \pm t_{0.005} s \sqrt{\frac{1}{n}+\frac{(x-\bar{X})^{2}}{\sum_{i=1}^{n}\left(x_{i}-\bar{X}\right)^{2}}}
$$

where:

- $t_{0.005}$ is the $t$-statistics value for a $99.9 \%$ confidence interval. We will use the $z$-statistics instead since $t_{0.005}=z_{0.005}=3.27$ for large samples of points (above 120);

- $n$ is the number of points;

- $s$ is the standard deviation from the expected value $\hat{y}$ at co-ordinate $x$ (see Thomas Wonnacott \& Ronald Wonnacott, 1990, p. 379):

$$
s=\sqrt{\frac{\sum_{i=1}^{n}\left(y_{i}-\hat{y}\right)}{n-2}}, \text { where } \hat{y}=a x+b
$$

- $\bar{X}$ is the average value of the various $x_{i}$ :

$$
\bar{X}=\frac{1}{n} \sum_{i=1}^{n} x_{i}
$$

\section{Evaluation}

We ran our alignment algorithm on the parallel texts of 10 language pairs as described in section 2.2. The table below summarises the results:

\begin{tabular}{crrrr}
\multicolumn{5}{c}{ Sub-corpus } \\
\hline Pair & Written Questions & \multicolumn{1}{c}{ Debates } & \multicolumn{1}{l}{ Judgements } & \multicolumn{1}{c}{ Average } \\
\hline pt-da & $128(5 \%)$ & $56(2 \%)$ & $114(35 \%)$ & $63(2 \%)$ \\
pt-de & $124(5 \%)$ & $99(2 \%)$ & $53(15 \%)$ & $102(3 \%)$ \\
pt-el & $118(5 \%)$ & $115(6 \%)$ & $60(20 \%)$ & $115(6 \%)$ \\
pt-en & $88(3 \%)$ & $102(4 \%)$ & $50(19 \%)$ & $101(4 \%)$ \\
pt-es & $59(1 \%)$ & $55(1 \%)$ & $143(21 \%)$ & $56(1 \%)$ \\
pt-fi & ---- & $60(26 \%)$ & $60(26 \%)$ \\
pt-fr & $148(5 \%)$ & $113(2 \%)$ & $212(49 \%)$ & $117(2 \%)$ \\
pt-it & $117(4 \%)$ & $104(2 \%)$ & $25(6 \%)$ & $105(2 \%)$ \\
pt-nl & $120(5 \%)$ & $73(1 \%)$ & $53(15 \%)$ & $77(2 \%)$ \\
pt-sv & ---- & - & $74(23 \%)$ & $74(23 \%)$ \\
\hline Average & $113(4 \%)$ & $90(2 \%)$ & $84(23 \%)$ & $92(2 \%)$ \\
\hline
\end{tabular}

Table 4: Average number of correspondence points in the first non-misalignment (average ratio of filtered and initial candidate correspondence points inside brackets).

On average, we end up with about $2 \%$ of the initial correspondence points which means that we are able to break a text in about 90 segments (ranging from 70 words to 12 pages per segment 
for the Debates). An average of just three filtrations are needed: the Histogram filter plus two filtrations with the Confidence Bands.

The figure below shows an example of a misaligning correspondence point.

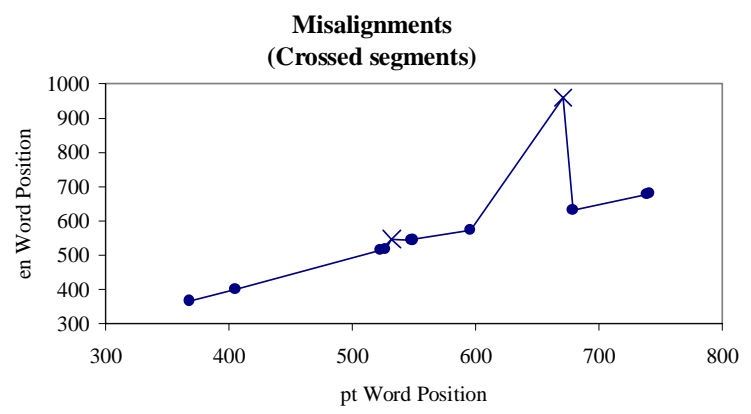

Figure 4: Bad correspondence points $(x-$ misaligning points; $\bullet$ - correspondence points).

Had we restricted ourselves to using homographs which occur only once (hapaxes), we would get about one third of the final points (António Ribeiro et al. 2000a). Hapaxes turn out to be good candidate correspondence points because they work like cognates that are not mistaken for others within the full text scope (Michel Simard and Pierre Plamondon, 1998). When they are in similar positions, they turn out to be reliable correspondence points.

To compare our results, we aligned the BAF Corpus (Michel Simard and Pierre Plamondon, 1998) which consists of a collection of parallel texts (Canadian Parliament Hansards, United Nations, literary, etc.).

\begin{tabular}{lrrrccc}
\multicolumn{5}{c}{ Equal Frequency Homographs } & \multicolumn{3}{c}{ BAF Analysis } \\
\hline Filename & \# Tokens & Segments & Chars / Segment & \# Segments & Chars / Segment & Ratio \\
\hline citi1.fr & 17556 & 49 & 1860 & 742 & 120 & $6,6 \%$ \\
citi2.fr & 33539 & 48 & 3360 & 1393 & 104 & $3,4 \%$ \\
cour.fr & 49616 & 101 & 2217 & 1377 & 140 & $7,3 \%$ \\
hans.fr & 82834 & 45 & 8932 & 3059 & 117 & $1,5 \%$ \\
ilo.fr & 210342 & 68 & 15654 & 7129 & 137 & $1,0 \%$ \\
onu.fr & 74402 & 27 & 14101 & 2559 & 132 & $1,1 \%$ \\
tao1.fr & 10506 & 52 & 1019 & 365 & 95 & $14,2 \%$ \\
tao2.fr & 9825 & 51 & 972 & 305 & 97 & $16,7 \%$ \\
tao3.fr & 4673 & 44 & 531 & 176 & 62 & $25,0 \%$ \\
verne.fr & 79858 & 29 & 12736 & 2521 & 127 & $1,2 \%$ \\
xerox.fr & 66605 & 114 & 2917 & 3454 & 85 & $3,3 \%$ \\
\hline Average & 111883 & 60 & 10271 & 3924 & 123 & $1,5 \%$ \\
\hline
\end{tabular}

Table 5: Comparison with the Jacal alignment (Michel Simard and Pierre Plamondon, 1998).

The table above shows that, on average, we got about $1.5 \%$ of the total segments, resulting in about $10 \mathrm{k}$ characters per segment. This number ranges from 25\% (average: 500 characters per segment) for a small text (tao3.fr-en) to $1 \%$ (average: $15 \mathrm{k}$ characters per segment) for a large text (ilo.fr-en). Although these are small num- bers, we should notice that, in contrast with Michel Simard and Pierre Plamondon (1998), we are not including:

- words defined as cognate "if their four first characters are identical";

- an 'isolation window' heuristics to reduce the search space;

- heuristics to define a search corridor to find candidate correspondence points;

We should stress again that the algorithm reported in this paper is purely statistical and recurs to no heuristics. Moreover, we did not reapply the algorithm to each aligned parallel segment which would result in finding more correspondence points and, consequently, further segmentation of the parallel texts. Besides, if we use the methodology presented in Joaquim da Silva et al. (1999) for extracting relevant string patterns, we are able to identify more statistically reliable cognates.

António Ribeiro and Gabriel Lopes (1999) report a higher number of segments using clusters of points. However, the algorithm does not assure $100 \%$ alignment precision and discards some good correspondence points which end up in bad clusters.

Our main critique to the use of heuristics is that though they may be intuitively quite acceptable and may significantly improve the results as seen with Jacal alignment for the BAF Corpus, they are just heuristics and cannot be theoretically explained by Statistics.

\section{Conclusions}

Confidence bands of linear regression lines help us to identify reliable correspondence points without using empirically found or statistically unsupported heuristics. This paper presents a purely statistical approach to the selection of candidate correspondence points for parallel texts alignment without recurring to heuristics as in previous work. The alignment is not restricted to sentence or paragraph level for which clearly delimited boundaries markers would be needed. It is made at whatever segment size as long as reliable correspondence points are found. This means that alignment can result at paragraph, sentence, phrase, term or word level.

Moreover, the methodology does not depend on the way candidate correspondence points are generated, i.e. although we used homographs with equal frequencies, we could have also boot- 
strapped the process using cognates (Michel Simard et al. 1992) or a small bilingual lexicon to identify equivalents of words or expressions (Dekai Wu 1994; Pascale Fung and Kathleen McKeown 1997; Melamed 1999). This is a particularly good strategy when it comes to distant languages like English and Chinese where the number of homographs is reduced. As António Ribeiro et al. (2000b) showed, these tokens account for about 5\% for small texts. Aligning languages with such different alphabets requires automatic methods to identify equivalents as Pascale Fung and Kathleen McKeown (1997) presented, increasing the number of candidate correspondence points at the beginning.

Selecting correspondence points improves the quality and reliability of parallel texts alignment. As this alignment algorithm is not restricted to paragraphs or sentences, $100 \%$ alignment precision may be degraded by language specific term order policies in small segments. On average, three filtrations proved enough to avoid crossed segments which are a result of misalignments. The method is language and character-set independent and does not assume any a priori language knowledge (namely, small bilingual lexicons), text tagging, well defined sentence or paragraph boundaries nor one-to-one translation of sentences.

\section{Future Work}

At the moment, we are working on alignment of sub-segments of parallel texts in order to find more correspondence points within each aligned segment in a recursive way. We are also planning to apply the method to large parallel Portuguese-Chinese texts. We believe we may significantly increase the number of segments we get in the end by using a more dynamic approach to the filtering using linear regression lines, by selecting candidate correspondence points at the same time that parallel texts tokens are input. This approach is similar to Melamed (1999) but, in contrast, it is statistically supported and uses no heuristics.

Another area for future experiments will use relevant strings of characters in parallel texts instead of using just homographs. For this purpose, we will apply a methodology described in Joaquim da Silva et al. (1999). This method was used to extract string patterns and it will help us to automatically extract 'real' cognates.

\section{Acknowledgements}

Our thanks go to the anonymous referees for their valuable comments on the paper. We would also like to thank Michel Simard for providing us the aligned BAF Corpus. This research was partially supported by a grant from Fundação para a Ciência e Tecnologia / Praxis XXI.

\section{References}

Peter Brown, Jennifer Lai and Robert Mercer (1991) Aligning Sentences in Parallel Corpora. In "Proceedings of the 29th Annual Meeting of the Association for Computational Linguistics", Berkeley, California, U.S.A., pp. 169-176.

Kenneth Church (1993) Char_align: A Program for Aligning Parallel Texts at the Character Level. In "Proceedings of the 31st Annual Meeting of the Association for Computational Linguistics", Columbus, Ohio, U.S.A., pp. 1-8.

Ido Dagan, Kenneth Church and William Gale (1993) Robust Word Alignment for Machine Aided Translation. In "Proceedings of the Workshop on Very Large Corpora: Academic and Industrial Perspectives", Columbus, Ohio, U.S.A., pp. 1-8.

ELRA (European Language Resources Association) (1997) Multilingual Corpora for Co-operation, Disk 2 of 2. Paris, France.

Pascale Fung and Kathleen McKeown (1994) Aligning Noisy Parallel Corpora across Language Groups: Word Pair Feature Matching by Dynamic Time Warping. In "Technology Partnerships for Crossing the Language Barrier: Proceedings of the First Conference of the Association for Machine Translation in the Americas", Columbia, Maryland, U.S.A., pp. 81-88.

Pascale Fung and Kathleen McKeown (1997) A Technical Word-and Term-Translation Aid Using Noisy Parallel Corpora across Language Groups. Machine Translation, 12/1-2 (Special issue), pp. 53-87.

William Gale and Kenneth Church (1991) A Program for Aligning Sentences in Bilingual Corpora. In "Proceedings of the 29th Annual Meeting of the Association for Computational Linguistics", Berkeley, California, U.S.A., pp. 177-184 (short version). Also (1993) Computational Linguistics, 19/1, pp. 75-102 (long version).

Martin Kay and Martin Röscheisen (1993) TextTranslation Alignment. Computational Linguistics, 19/1, pp. 121-142.

Samuel Kotz, Norman Johnson and Campbell Read (1982) Encyclopaedia of Statistical Sciences. John Wiley \& Sons, New York Chichester Brisbane Toronto Singapore. 
I. Dan Melamed (1999) Bitext Maps and Alignment via Pattern Recognition. Computational Linguistics, 25/1, pp. 107-130.

António Ribeiro, Gabriel Lopes and João Mexia (2000a) Using Confidence Bands for Alignment with Hapaxes. In "Proceedings of the International Conference on Artificial Intelligence (IC'AI 2000)", Computer Science Research, Education and Applications Press, U.S.A., volume II, pp. 1089-1095.

António Ribeiro, Gabriel Lopes and João Mexia (2000b, in press) Aligning Portuguese and Chinese Parallel Texts Using Confidence Bands. In "Proceedings of the Sixth Pacific Rim International Conference on Artificial Intelligence (PRICAI 2000) - Lecture Notes in Artificial Intelligence", Springer-Verlag.

Joaquim da Silva, Gaël Dias, Sylvie Guilloré, José Lopes (1999) Using Localmaxs Algorithms for the Extraction of Contiguous and Non-contiguous Multiword Lexical Units. In Pedro Barahona and José Alferes, eds., "Progress in Artificial Intelligence - Lecture Notes in Artificial Intelligence", number 1695, Springer-Verlag, Berlin, Germany, pp. 113-132.

Michel Simard, George Foster and Pierre Isabelle (1992) Using Cognates to Align Sentences in Bilingual Corpora. In "Proceedings of the Fourth International Conference on Theoretical and Methodological Issues in Machine Translation TMI-92”, Montreal, Canada, pp. 67-81.

Michel Simard and Pierre Plamondon (1998) Bilingual Sentence Alignment: Balancing Robustness and Accuracy. Machine Translation, 13/1, pp. 59-80.

Dekai Wu (1994) Aligning a Parallel English-Chinese Corpus Statistically with Lexical Criteria. In "Proceedings of the 32nd Annual Conference of the Association for Computational Linguistics", Las Cruces, New Mexico, U.S.A., pp. 80-87.

Thomas Wonnacott and Ronald Wonnacott (1990) Introductory Statistics. 5th edition, John Wiley \& Sons, New York Chichester Brisbane Toronto Singapore, $711 \mathrm{p}$. . 